\title{
The Pendulum: drugs and pain and persons with spinal cord injuries and disorders
}

\author{
Marcalee Alexander ${ }^{1}$
}

Received: 2 July 2018 / Accepted: 4 July 2018

(C) International Spinal Cord Society 2018

If you regularly read Spinal Cord Series and Cases, you will be aware that spinal cord injury (SCI) causes neuropathic and other chronic pain syndromes that can cause significant quality of life issues for persons with SCI. Often, pain can be more troubling than the disability associated with injury. As in other areas, over the past 30 years, we have experienced a shift in the pendulum for caring for individuals with SCI from recommendations to never use opioids as treatment of chronic pain after SCI to the common use of opioids for individuals with tetraplegia or paraplegia.

At present, we are constantly barraged with new information regarding the "opioid crisis" and the number of individuals provided with opioids who have become addicted or are at risk of overdose. While discussion of this issue has started with the ambulatory chronic pain population, this issue is also important, but less well addressed in the population of persons with SCI.

Similarly, the legality of the recreational and medicinal use of cannabis is now expanding and acknowledged. This is also an area of importance, but one that is relatively ignored for study for individuals with SCIs.

In order to begin the dialogue and raise the issues regarding opioids and cannabis use for individuals with SCIs, I have invited two groups to discuss these topics. New, Bryce, and Kertesz raise important issues regarding opioids. Are they beneficial? Should persons with SCIs already on opioids be taken off? Should new individuals be given the option for opioids? These are important issues for us to consider; yet, they are difficult to research. Similarly, how can we best determine the effects of components of medical cannabis for individuals with SCIs? Could the sensory experiences associated with the use of cannabis be beneficial for individuals with SCIs? What do we need to do to compare the effects of cannabis with opioids for pain after SCI? Are there more benefits or complications associated with one form of management versus another? And could either of these therapies be superior to other accepted treatments?

To accompany this topic there will also be pulse surveys circulating through ISCOS regarding pain management. We want to start the dialogue and have you share your thoughts. Please read the point counterpoint articles in this issue and send your letters to the editor addressing these issues, and please when you get an opportunity to complete the pulse surveys, do so. There is one specifically for prescribers that can be accessed at https://redcap.mountsinai.org/redcap/ surveys/?s=3JNJXHPCW3 and the other for all SCI team members that can be accessed at https://www. surveymonkey.com/r/YFYLN8K

Thanks again to all of you for reading the journal and participating in the surveys. Remember this is your journal and your opinion counts!

Marcalee Alexander, MD Editor-in-Chief, SCSC
Marcalee Alexander

spinalcordmd@live.com

1 University of Alabama at Birmingham School of Medicine, Birmingham, AL 35233, USA 